Befragten - finanzielle Solidarität innerhalb der EU ablehnen. Wie die Kontroversen innerhalb der CDU/CSU und der FDP in Deutschland zeigen, ist hier ein beachtliches Mobilisierungspotenzial für euro-nationalistische Positionen entstanden, die in einigen Ländern der Euro-Zone bereits im Parteienspektrum vertreten sind. Auch in Deutschland ist Platz für eine euro-nationalistische rechtspopulistische Partei.

Ich behaupte also, dass die Politisierung europapolitischer Fragen weiter zunehmen wird, und zwar entlang der Spaltung zwischen Anhängern eines modernen und weltoffenen Europa einerseits und Vertretern eines nationalistischen und nach innen gewandten Europa andererseits. Die politischen Eliten sind auf diesen Konflikt kaum vorbereitet und fangen nur sehr zögerlich an, ihre Vision der europäischen Integration offensiv zu verteidigen. Dabei geht es in Zukunft nicht mehr um die Frage „Bist Du für oder gegen die EU?", sondern darum, welche Politik in der EU betrieben werden soll. Diese Schlussfolgerung meines Buches bleibt auch 2012 richtig.

Thomas Risse

\section{Politische Theorie und Ideengeschichte}

Geuss, Raymond. Kritik der politischen

Philosophie. Eine Streitschrift. Ham-

burg. Hamburger Edition. 2011. 141

Seiten. 12,00€.

Raymond Geuss hat unter dem Titel Kritik der politischen Philosophie eine schmale, aber gewichtige Streitschrift vorgelegt. Deren Ziel besteht in nichts Geringerem, als den Versuch einer Neubestimmung der grundsätzlichen
Ausrichtung von politischer Theorie und Philosophie zu unternehmen. Geuss wählt, wie der Untertitel bereits festhält, zur Annäherung an dieses Ziel den Weg des offensiven Streits. Sein Unbehagen entzündet sich an der Beobachtung, dass immer größere Teile der politischen Philosophie der Gegenwart als Ethik, die auf den Bereich der Politik angewendet wird, betrieben werden. Geuss hält nicht lange mit seinem Urteil hinter dem Berg, sondern notiert von Anfang an deutlich, dass dies aus seiner Perspektive eine für die politische Philosophie und Theorie im Ganzen fatale Fehlentwicklung darstellt. Damit attackiert Geuss im Grunde die gesamte Entwicklung der politischen Philosophie, wie sie sich seit dem Erscheinen von Rawls' A Theory of Justice 1971 vollzogen hat und wie sie in Deutschland neben der Orientierung an Rawls auch im Anschluss an Habermas' Faktizität und Geltung (1992) betrieben wird, frontal. Der nach seiner Einschätzung vorherrschende kantianische Strang der politischen Philosophie legt sich auf ein bestimmtes Verhältnis von politischer Philosophie und Normativität fest, das Geuss als die „'Ethik-hat-Vorrang'-Lesart“ (11) des Verhältnisses von Politik und Normativität bezeichnet. Diese Lesart postuliert im Unterschied zu der aus Geuss' Sicht unkontroversen allgemeinen These, dass Politik „kein streng wertfreies Unternehmen sein kann" (ibid.) eine hierarchische Beziehung von Ethik bzw. Moralphilosophie und Politik, in deren Kern die Vorstellung steht, dass es begründete ethische Prinzipien sind, über deren Anwendung im Feld der Politik nachzudenken die Aufgabe der politischen Philosophie ausmacht. Kennzeichnend für diese Version der Ethik, die im deutschsprachigen Raum eher 
unter dem Begriff der Moraltheorie verhandelt wird, sind auf der begründungstheoretischen Ebene „empirische Abstinenz und Systematik" (20). Nach Geuss' Überzeugung sind solche Fassungen der politischen Philosophie in doppelter Hinsicht verfehlt: Erstens mangelt es ihnen, bedingt durch den Versuch, allgemeine Prinzipien zu begründen, an einer hinreichenden historischen Kontextualisierung. Zweitens, und ungleich schwerer wiegend, verlieren sie zudem ihren Gegenstand, die Politik, aus den Augen, weil der von vorne herein normativistisch eingefärbte Blick jenes Maß an Realismus unmöglich macht, das nach seiner Überzeugung unbedingt nötig ist, um das eigentlich Politische der Politik zu erfassen. Kantianische Zugänge zur politischen Philosophie verlieren die Machtbezogenheit von Politik aus den Augen, so sucht uns Geuss in einer auch vor polemischen Zuspitzungen nicht zurückschreckenden Form zu überzeugen, weil sie „Opfer eines Fetischismus" (32) werden, der unablässig nach der Begründung normativer Prinzipien spürt und dabei deren Bedeutung im Reich der Politik hoffnungslos überschätzt. Diese Machtvergessenheit identifiziert Geuss folgerichtig auch als einen der Kardinalfehler von Rawls' politischer Philosophie (123ff.). Nach Geuss' eigener Auffassung muss Politik hingegen theoretisch mithilfe einer Erweiterung von Lenins Frage „Wer wen?“ (41) untersucht werden, die dann lautet „Wer ,tut' wem was zu wessen Nutzen?" (ibid.). Erst durch diese Frage kann nach seiner Auffassung die Prinzipienbegründungsfixierung aufgebrochen werden. Was dann in den Blick gerät, umschreibt Geuss mithilfe dreier Substantive, deren Beziehungstrias zugleich auch sein Poli- tikverständnis umreißt: „Politisch zu denken heißt über Handlungsfähigkeit, Macht, Interessen und deren Verhältnis untereinander nachzudenken " (ibid.). Radikal ist Geuss in der Ausweitung des Anwendungsbereichs dieses Politikbegriffs: Nicht nur in der sogenannten Realpolitik soll er Gültigkeit besitzen, sondern er soll auch Aufschluss geben über die Genese von politischen Theorien. Deshalb spricht Geuss hier von einer „Politik der Theoriebildung“ (46) und weist spätestens damit Aspirationen auf eine universelle Begründung normativer Prinzipien ganz grundsätzlich zurück.

Mit diesem Politikverständnis positioniert sich der Autor übrigens der Sache nach in der Nähe eines zum Kantianismus alternativen Strangs der zeitgenössischen politischen Philosophie, der von den Machtanalysen Michel Foucaults geprägt ist. Eng verwandt mit der foucaultschen Unternehmung ist denn auch ein weiterer wesentlicher Aspekt von Geuss' Schrift, der die Beziehung zur Kritik betrifft. Geuss weist im Abschlusskapitel (129-137) ganz entschieden eine Forderung zurück, die ein Kennzeichnen kantianischer politischer Theorien ist und die im gegenwärtigen Theoriediskurs nachgerade dominant geworden ist: Nach seiner Auffassung ist das Gebot, positive Schlüsse ziehen zu können, wenn man sich an die Aufgabe einer Kritik bestehender Verhältnisse macht, geradezu der Sargnagel einer vehementen Kritik. Lässt man sich auf das Postulat ein, bei aller Kritik konstruktiv zu sein, dann wird diese Konstruktivität unversehens zum Prüfstein der Berechtigung der Kritik: Kritisieren dürfte demnach nur, wer „eine positive Alternative [...] parat hat" (129). Es ist diese Konsequenz, auf die Geuss' Kritik der kantianisch geprägten 
politischen Philosophie zuläuft und sie ist es auch, die diese kleine Schrift zu einem so wichtigen Beitrag in der aktuellen Auseinandersetzung über das angemessene Verständnis von politischer Theorie bzw. Philosophie und Kritik macht: Indem Geuss eine ideologiekritische, realistische Machtanalyse vorschlägt (75-81), die keineswegs die Beziehung des Politischen zur Normativität dementiert, die aber energisch bestreitet, dass Normativitätsbegründung das Kerngeschäft einer kritischen politischen Philosophie und Theorie ausmacht, unternimmt er einen schlagkräftigen Versuch, einer Form kritischen politischen Denkens jenseits des kantianischen Begründungsgebots den Weg zu bahnen.

Oliver Flügel-Martinsen

\section{Honneth, Axel. Das Ich im Wir. Studien} zur Anerkennungstheorie. Berlin. Suhrkamp 2010. 308 Seiten. $12 €$.

An der Umwandlung der „Anerkennung " von einem Gegenstand oder einem Themenfeld sozialwissenschaftlicher oder sozialphilosophischer Forschung in den Namen einer eigenständigen theoretischen Richtung hat neben anderen Axel Honneth erheblichen Anteil. Er legt nun ergänzende, klärende und vertiefende Untersuchungen vor, die den explikativen Anspruch der Anerkennungstheorie weiter untermauern sollen. In vier Abschnitten unternimmt Honneth dies a) über die Rückvergewisserung in der Hegelschen Philosophie des Geistes, der er die strukturellen Schemata seiner Theorie entlehnt, b) über systematische Auseinandersetzungen mit gerechtigkeitstheoretischen Problemen und Positionen, c) über soziologisch-deskriptive Analysen und d) über psychoanalytische Ergänzungen.

Als Leitfaden der untereinander methodisch wie thematisch recht heterogenen Beiträgen wird man die theoriepolitische These ausmachen können, dass eine Umstellung der sozialphilosophischen Theoriebildung von der Orientierung an Gleichheit auf die an Anerkennung eine Wende von monistischen auf pluralistische Ansätze bedeutet (167). Vorbereitet wird diese These in den beiden Hegel-Aufsätzen, die der bereits bekannten Verwendung, die Honneth von Hegel macht, nicht viel Neues hinzufügen; entfaltet wird sie vor allem in dem programmatischen Aufsatz „Das Gewebe der Gerechtigkeit. Über die Grenzen des zeitgenössischen Prozeduralismus“. In den gegenwärtig dominanten Gerechtigkeitstheorien, die ihre liberale Stoßrichtung vor allem einem kantischen Erbe verdanken, werden zunächst drei relevante Bestandteile kritisch gesichtet: ein prozeduralistisches Grundschema, die Orientierung an der Verteilungsgerechtigkeit und ihre Staatsfixierung. Der Ausgangspunkt bei der Überzeugung, Gerechtigkeit werde in erster Linie über die Gewährung eines allen gemeinsamen Spielraums für die Verfolgung individueller Präferenzen gewährleistet, sorgt dafür, dass die drei Aspekte dort an ihre Grenzen kommen, wo das individuelle Nutzenkalkül das Gerechtigkeitsempfinden nicht zur Gänze abzudecken vermag beziehungsweise mit diesem in Widerspruch gerät. So muss der Prozeduralismus Autonomie schon voraussetzen, obwohl sie doch erst am Ende des Abgleichs von Interessen als Resultat sich ergeben soll. So schließt das Distributionsparadigma all jene Aspekte der Gerechtigkeit aus der Betrachtung aus, die sich 